\title{
Temperatura basal, duração do ciclo e constante térmica para a cultura do crambe
}

\author{
Felipe Gustavo Pilau $\left({ }^{1 *}\right)$; Rafael Battisti $\left({ }^{2}\right)$, Lucindo Somavilla $\left({ }^{3}\right)$, Luciano Schwerz $\left({ }^{4}\right)$ \\ (') Universidade Federal de Santa Maria, Departamento de Fitotecnia, Av. Roraima n. ${ }^{1000, ~ 97105-900 ~ S a n t a ~ M a r i a ~(R S), ~ B r a s i l . ~}$ \\ (2) Universidade de São Paulo, Escola Superior de Agricultura Luiz de Queiroz, Programa de Pós-graduação em Engenharia de \\ Sistemas Agrícolas. Piracicaba (SP), Brasil. \\ (3) Universidade Federal de Santa Maria, Frederico Westphalen (RS), Brasil. \\ (4) Universidade Federal de Santa Maria, Programa de Pós-graduação em Agronomia, Agricultura e Ambiente, Frederico Westphalen \\ (RS), Brasil. \\ (*) Autor correspondente: fgpilau@smail.ufsm.br
}

Recebido: 4/abr./2011; Aceito: 11/ago./2011

\begin{abstract}
Resumo
A cultura do crambe surge como alternativa de produção, destacando sua precocidade e o potencial para a produção de óleo, em estação fria. A escassez de informações sobre parâmetros climáticos e fenológicos conduziu à determinação da temperatura basal inferior, da duração do ciclo e da constante térmica da cultura do crambe em Frederico Westphalen (RS). O trabalho foi composto por sete épocas de semeadura em 2009 e quatro em 2010. A temperatura basal inferior foi determinada pelos métodos do menor desvio-padrão e do desenvolvimento relativo. A temperatura basal para o subperíodo emergência-maturação (EME-MAT) foi de 9,5 C, enquanto para os subperíodos emergência-início da floração (EME-IFLO), emergência-fim da floração (EME-FFLO) e início da floração-fim da floração (IFLO-FFLO) foram, respectivamente, de 10,2 •C, $10,8^{\circ} \mathrm{C}$ e 10,0 ${ }^{\circ} \mathrm{C}$, obtidas pelo método do menor desvio-padrão. O subperíodo compreendido entre EME-MAT variou de 74 a 136 dias, com duração média de 96 dias. Para os subperíodos EME-IFLO, EME-FFLO e IFLO-FFLO a duração média foi, respectivamente, de 52, 82 e 29 dias. Considerando-se os valores de temperatura basal para cada subperíodo, a duração média entre EME-MAT foi de $691^{\circ} \mathrm{C}$ dia, variando entre $576{ }^{\circ} \mathrm{C}$ dia e $792^{\circ} \mathrm{C}$ dia. Para os subperíodos EME-IFLO, EME-FFLO e IFLO-FFLO, a duração média do foi de $279{ }^{\circ} \mathrm{C}$ dia; $444^{\circ} \mathrm{C}$ dia e $220^{\circ} \mathrm{C}$ dia, respectivamente.
\end{abstract}

Palavras-chave: Crambe abyssinica Hochst., temperatura, fenologia.

\section{Basal temperature, crop growth cycle duration and growing degree-days of crambe}

\section{Abstract}

Crambe is an alternative crop, precocious and with potential to produce oil during the cold season. The scarcity of information about climatic and phenological parameters led to the determination of basal temperature, crop growth cycle duration and growing degree-days of Crambe. The research was composed of seven sowing dates in 2009 and four in 2010. Basal temperature was determined by the methods of least variability and relative development. The basal temperature for the subperiod emergence-maturation (EME-MAT) was $9.5^{\circ} \mathrm{C}$, whereas for the subperiods seedling emergency-early flowering stage (EME-IFLO), seedling emergency-end of flowering (EME-FFLO) and early flowering-end of flowering (IFLO-FFLO) were $10.2^{\circ} \mathrm{C}, 10.8^{\circ} \mathrm{C}$ and $10.0{ }^{\circ} \mathrm{C}$, respectively. The subperiod between EME-MAT ranged from 74 to 136 days with an average duration of 96 days. For EME-FLO, EME-FFLO and IFLO- FFLO the average duration was 52, 82 and 29 days, respectively. Considering the values of basal temperature for each period or subperiod, the average time between EME-MAT was $691{ }^{\circ} \mathrm{C}$ day, ranging between $576{ }^{\circ} \mathrm{C}$ day and $792^{\circ} \mathrm{C}$ day. For EME-IFLO, EME-FFLO and IFLO-FFLO, the average was $279{ }^{\circ} \mathrm{C}$ day, varying between $444^{\circ} \mathrm{C}$ day and $220^{\circ} \mathrm{C}$ day.

Key words: Crambe abyssinica Hochst., temperature, phenology. 


\section{INTRODUÇÃO}

Pertencente à família das crucíferas, nativo da zona Mediterrânea, a cultura do crambe (Crambe abyssinica Hochst.) surge com potencial para a produção de óleo destinado à fabricação de biodiesel em estação fria, energia limpa e renovável, em substituição aos combustíveis fósseis, auxiliando na contenção da emissão de gases de efeito estufa. É uma cultura oleaginosa capaz de tolerar temperaturas de até $-6,0{ }^{\circ} \mathrm{C}$ por algumas horas sem danos significantes (Fowler, 1991). Durante o período vegetativo necessita temperaturas entre $15^{\circ} \mathrm{Ce} 25^{\circ} \mathrm{C}$ (FALAsCA et al., 2010)

Dentre as principais características da cultura está sua precocidade, com grãos maduros aos 90/100 dias, boa produtividade, podendo atingir $2.500 \mathrm{~kg} \mathrm{ha}^{-1}$, adaptação a variadas condiçốes pedoclimáticas (LAZZERI et al., 1995; Lessman e Meier, 1972; White e Higgins, 1966) e maturação uniforme, facilitando a colheita mecanizada (FAlasca et al., 2010).

No Brasil, o cultivo do crambe foi iniciado em 1995, no Estado do Mato Grosso do Sul, com recentes áreas plantadas no Estado do Paraná. Por esse motivo, os estudos no país são restritos e a busca de informações pode auxiliar no desenvolvimento produtivo. Entre as várias informaçóes necessárias, a análise do desenvolvimento pode explicar satisfatoriamente o efeito da temperatura do ar sobre essa espécie e sua adaptação a novas áreas de cultivo.

No Estado de Dakota do Norte, EUA, onde o Crambe é economicamente explorado desde 1990 (KNights, 2002), a cultura tem um subperíodo de produção, da semeadura à maturação, de 85 a 100 dias (Golz, 1993). Já em avaliaçốes no Estado do Arizona, submetido a diferentes épocas de semeadura no outono, em dois anos agrícolas, apenas o subperíodo da semeadura à floração teve duração de 79 a 100 dias, e o florescimento levou de 22 a 39 dias (Adamsen e Coffelt, 2005).

Para avaliar o efeito da temperatura do ar, pode-se utilizar o conceito de soma térmica ou graus-dia, que se baseia na resposta da planta à temperatura do ar, destacando a existência de temperaturas basais que controlam o crescimento e desenvolvimento da cultura. Mesmo sob condição hídrica e níveis de radiação solar ideais, a planta não se desenvolve e seu crescimento é extremamente reduzido, quando submetida à temperatura inferior a um valor mínimo, ou superior a um valor máximo, com exigência específica de temperatura do ar para cada fase fenológica de desenvolvimento (Went, 1953; Monteith e Elston, 1996).

Segundo Gilmore e Rogers (1958), a soma térmica é um índice amplamente utilizado por ser de simples execução e expressar uma das melhores medidas de tempo biológico das plantas. A soma térmica ou constante térmica é obtida a partir da determinação da temperatura basal, geralmente inferior, pois a superior dificilmente é atingida. Posteriormente, obtém-se a soma térmica do ciclo em função da temperatura do ar, possibilitando através destas informaçôes a realizaçáo do planejamento da duração do ciclo, obtendo-se as melhores épocas de semeadura para locais onde o cultivo não é realizado, mas se dispóe de informaçôes climáticas.

Para a cultura do crambe, são necessários aproximadamente $1350^{\circ} \mathrm{C}$ dia acumulados entre a semeadura e a maturação fisiológica (KMEC et al., 1998), e temperatura basal inferior igual a $2,5^{\circ} \mathrm{C}$. Toebe et al. (2010), com o mesmo valor de temperatura basal inferior destacado por KMEC et al. (1998), obtiveram de $1165,3{ }^{\circ} \mathrm{C}$ dia a $1175,8^{\circ} \mathrm{C}$ dia entre emergência e senescência. Para a cultura da canola, outra crucífera, semeada em mesma época, MORRISON et al. (1989) relatam uma temperatura base inferior (cv. Westar), próxima a $5^{\circ} \mathrm{C}$.

Desta forma, diante da importância que a cultura do crambe poderá ter na produção agrícola brasileira e da escassez de parâmetros climáticos e fenológicos que auxiliem no planejamento e manejo da cultura, o objetivo deste trabalho foi determinar a temperatura basal inferior, duração do ciclo de desenvolvimento e constante térmica da cultura do crambe.

\section{MATERIAL E MÉTOdOS}

O experimento foi desenvolvido no município de Frederico

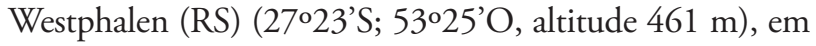
solo do tipo Latossolo Vermelho Distrófico (Embrapa, 1999), e clima subtropical, com verão quente ( $\mathrm{CFa}$ ), conforme classificação de Köppen (Moreno, 1961).

Para adubação, em semeadura, foram utilizados $150 \mathrm{~kg} \mathrm{ha}^{-1}$ da formula 5-20-10 (NPK), acrescidos de $25 \mathrm{~kg} \mathrm{ha}^{-1}$ de nitrogênio, na forma de uréia, quando as plantas estavam com quatro folhas expandidas, conforme recomendaçáo de adubaçáo e calagem para a cultura da canola (Tомм et al., 2009).

Os tratamentos foram compostos por sete épocas de semeadura em 2009 e quatro em 2010, sendo: 13/5/2009, 28/5/2009, 11/6/2009, 14/7/2009, 31/7/2009, 17/8/2009, 3/9/2009, 23/6/2010, 8/7/2010, 29/7/2010 e 29/8/2010, utilizando a cultivar de crambe FMS Brilhante. A semeadura foi realizada em linhas, espaçadas em 0,17 m, com estande final de 100 plantas $\mathrm{m}^{-2}$, dentro de parcelas de $12 \mathrm{~m}^{2}$ ( $3 \mathrm{~m} \mathrm{x} 4 \mathrm{~m}$ ). Para cada época foram instaladas quatro repetiçóes.

A temperatura do ar foi medida, em escala horária, na estação meteorológica automática do Instituto Nacional de Meteorologia, localizada a aproximadamente 100 metros da área experimental.

Assumiu-se como data de emergência (EME) o momento em que $50 \%$ das plantas estavam com cotilédones abertos acima do nível do solo; o início de floração (IFLO) quando em $50 \%$ das plantas havia pelo menos uma flor aberta; o fim de floração (FFLO) o momento em 
que as plantas não tinham mais flores e a maturação fisiológica (MAT) quando em $50 \%$ das cápsulas a coloração era amarronzada.

Para a determinação da temperatura basal inferior para o subperíodo de desenvolvimento EME-MAT, e subperíodos EME-IFLO, EME-FFLO e IFLO-FFLO, foram utilizados os métodos do menor desvio-padrão (ARNOLD, 1959), e a combinação dos métodos do desenvolvimento relativo (DR) e de regressão linear (BRUNini et al., 1976; GBur et al., 1979).

Para o método do menor desvio-padrão atribuiu-se a priori temperaturas basais inferiores, variando em escala unitária, de $0{ }^{\circ} \mathrm{C}$ a $13{ }^{\circ} \mathrm{C}$. Posteriormente determinou-se o desvio-padrão (Sdd) em graus-dias para cada temperatura, considerando como temperatura basal inferior a de menor desvio-padrão em dias $(\mathrm{Sd})$, conforme a equação a seguir:

$$
\mathrm{Sd}=\mathrm{Sdd} /(\mathrm{Xt}-\mathrm{Tb})
$$

em que, Xt é a temperatura média do ar $\left({ }^{\circ} \mathrm{C}\right)$ para todas as épocas de semeadura e $\mathrm{Tb}$ a temperatura basal inferior escolhida a priori.

$\mathrm{Na}$ avaliação do método do desenvolvimento relativo (DR) utilizaram-se as equaçóes:

$$
\begin{gathered}
\mathrm{DR}=\mathrm{b} \cdot \text { Tmed }+\mathrm{a} \\
\mathrm{DR}=100 / \mathrm{n} \\
\mathrm{Tb}=-\mathrm{a} / \mathrm{b}
\end{gathered}
$$

em que Tmed é a temperatura média do ar $\left({ }^{\circ} \mathrm{C}\right)$ do período, n é a duração do período em dias, a e b são os coeficientes linear e angular da regressáo linear simples obtidos na relação entre DR e Tmed.

Em seguida, determinou-se a constante térmica de cada fase de desenvolvimento, obtida pela equação:

$$
\mathrm{GD}=(\text { Tmed-Tbv }){ }^{*} \mathrm{n}
$$

em que GD é constante térmica em cada fase de desenvolvimento e $\mathrm{Tb}_{\mathrm{v}}$, temperatura basal inferior encontrada pelo método da menor variabilidade.

Com análise de regressão linear foram obtidos modelos de estimativa para o subperíodo EME-MAT, e subperíodos EME-IFLO, EME-FFLO e IFLO-FFLO, a partir da temperatura média do ar.

\section{RESULTADOS E DISCUSSÃO}

Para o subperíodo emergência-maturação (EME-MAT), observou-se que o menor desvio-padráo foi obtido para a
Tbi de $9,5^{\circ} \mathrm{C}$, enquanto para os subperíodos emergênciainício da floração (EME-IFLO), emergência-fim da floração (EME-FFLO) e início da floração-fim da floração (IFLO-FFLO), os valores de Tbi foram, respectivamente, de $10,2^{\circ} \mathrm{C}, 10,8^{\circ} \mathrm{C}$ e $10,0^{\circ} \mathrm{C}$ (Figura 1). Diferenças nos valores de $\mathrm{Tb}$ de distintos subperíodos são comumente verificadas nas mais variadas espécies e materiais genéticos (Streck et al., 2003; Pedro Júnior et al., 2004; Pezzopane et al., 2008; Souza et al., 2009).

A partir do método do desenvolvimento relativo (DR) (Figura 2), observaram-se valores de Tb ligeiramente diferentes daqueles obtidos pelo método do menor desvio-padrão, iguais a $8,9^{\circ} \mathrm{C}$ para EME-MAT, 9,6 ${ }^{\circ} \mathrm{C}$ para EME-IFLO, $10,1^{\circ} \mathrm{C}$ para EME-FFLO e de $9,2^{\circ} \mathrm{C}$ para IFLO-FFLO, indicando que os métodos de estimativa são

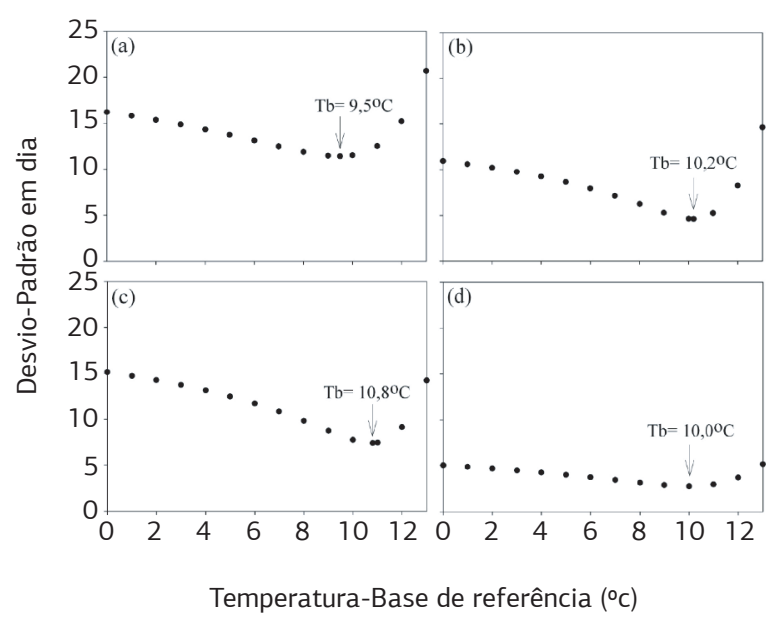

Figura 1. Temperatura basal inferior nas fases de desenvolvimento: (a) EME-MAT; (b) EME-IFLO; (c) EME-FFLO; (d) IFLO-FFLO, obtidas pelo método do menor desvio-padráo para a cultura do crambe.

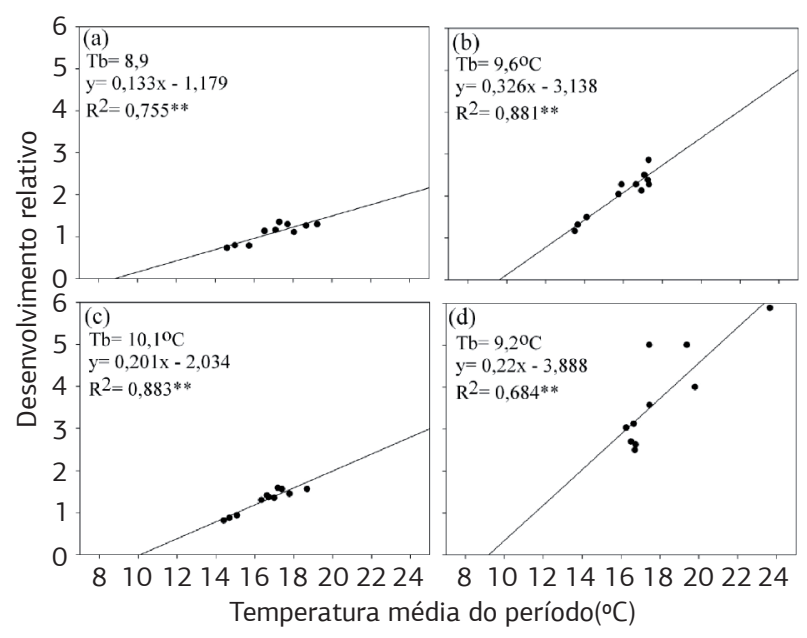

Figura 2. Temperatura basal inferior, para as fases de desenvolvimento: (a) EME-MAT; (b) EME-IFLO; (c) EMEFFLO; (d) IFLO-FFLO, obtida pelo método de desenvolvimento relativo para a cultura do crambe. ${ }^{* *}$ : Significativo pelo teste F a 1\% de probabilidade. 
de eficiência semelhante para determinação da temperatura basal inferior do crambe.

Determinando a temperatura basal inferior para três cultivares de triticale, com base nos mesmos métodos adotados no trabalho, Pedro Junior et al. (2004) obtiveram valores de $\mathrm{Tb}$ próximos, entre $8^{\circ} \mathrm{C}$ e $10,4^{\circ} \mathrm{C}$, porém, de forma contrária, os maiores valores de $\mathrm{Tb}$ foram apontados pelo método do DR. Da mesma forma, Müller et al. (2009) observou baixa variaçáo, de 0,1 a $0,7^{\circ} \mathrm{C}$, nos valores de $\mathrm{Tb}$ obtidos pelos dois métodos para a cultura do azevém.

Para a cultura da canola, espécie da mesma família, a temperatura basal inferior de $5{ }^{\circ} \mathrm{C}$ é comumente adotada para quantificação da soma térmica (Morrison et al.,1989; DALMAGo et al., 2008). A diferença entre as temperaturas basais observadas para o período EME-MAT do crambe, de $9,5^{\circ} \mathrm{C}$ e $8,9^{\circ} \mathrm{C}$, com a referida $\mathrm{Tb}$ da cultura da canola, refletem-se na resistência a baixas temperaturas. Enquanto plantas jovens de crambe são severamente danificadas a $-1{ }^{\circ} \mathrm{C}$, de forma geral, plântulas de canola, em estádio cotiledonar, morrem sob temperatura de $-3{ }^{\circ} \mathrm{C}$ e $-4^{\circ} \mathrm{C}$. Sob não aclimatação, plantas de canola morrem rapidamente a $-4,6{ }^{\circ} \mathrm{C}$, enquanto plantas com cinco ou mais folhas, crescendo em ambiente com temperatura média do ar inferior a $18{ }^{\circ} \mathrm{C}$ resistem a geadas de até $-8,6{ }^{\circ} \mathrm{C}$ (RAPACZ, 1999; Hawkins et al., 2002).

Com relação aos trabalhos de Toebe et al. (2010), que para estimar o plastocrono em Crambe adotou $\mathrm{Tb}$ de $2,5^{\circ} \mathrm{C}$, recomendada por KMEC et al. (1998), e de Adamsen e Coffelt (2005) cuja $\mathrm{Tb}$ de $0{ }^{\circ} \mathrm{C}$ foi adotada para avaliação de desenvolvimento do crambe no Estado do Arizona, Estados Unidos, também semelhante ao utilizado por Meijer e Mathijssen (1996), os valores de Tb obtidos nesse trabalho pelos métodos do menor desvio-padrão $(\mathrm{Sd})$ e desenvolvimento relativo (DR) foram no mínimo $6,4^{\circ} \mathrm{C}$ superiores. Meijer e Mathijssen (1996) propóem uma temperatura basal para expansão da folha de crambe de $6,8^{\circ} \mathrm{C}$, expressivamente mais próximos aos valores obtidos.

As diferenças entre a Tbi obtidas neste trabalho e as referidas na literatura podem estar associadas às condiçóes locais de cultivo, como o fotoperíodo (ADAMSEN e Coffelt, 2005), além das características da espécie e da cultivar. FALASCA et al. (2010) também comenta que a cultura do crambe pode ser considerada uma espécie de primavera ou inverno, dependendo do regime térmico da região onde está implantada.

Para o subperíodo compreendido entre EME e MAT, a variação entre as datas de semeadura foi de 74 a 136 dias, com duração média de 96 dias. Já para os subperíodos EME-IFLO, EME-FFLO e IFLO-FFLO, a duração média foi, respectivamente, de 52, 82 e 29 dias (Tabela 1). Adamsen e Coffelt (2005) obtiveram valores de 74 a 104 dias entre a semeadura e o início da floração para crambe (cv. Meyer), no Estado do Arizona, EUA, próximos aos 86 e 76 dias da EME-IFLO obtidos paras as datas de semeadura de 13/5/2009 e 28/5/2009.
Esses resultados associam-se a temperaturas médias mais baixas ao longo de todo o ciclo, de $15,2{ }^{\circ} \mathrm{C}$ para 13/5/2009 e $15,0^{\circ} \mathrm{C}$ para $28 / 5 / 2009$ e, principalmente, aos valores de temperatura média entre EME e IFLO, de $13,9^{\circ} \mathrm{C}$ para a primeira época de semeadura, e de $14,1^{\circ} \mathrm{C}$ para a segunda época de semeadura, período em que a cultura teve sua folhagem parcialmente danificada pela ocorrência de geada, com temperatura mínima de $-2,7^{\circ} \mathrm{C}$ (Tabela 1). Quanto aos períodos de floraçáo (IFLO-FFLO), AdAmsen e Coffelt (2005) destacam variaçôes de 22 até 49 dias, novamente similares aos observados nesse trabalho (Tabela 1 ).

Para a Argentina, ao simular um zoneamento de aptidão agrícola para a cultura do Crambe, FALASCA et al. (2010) destacam que para cultivo de primavera, semeado em outubro, a colheita ocorre no prazo de 90 a 100 dias após a emergência, também similar aos resultados verificados para semeaduras realizadas mais tardiamente na estaçáo de inverno - em agosto e setembro (Tabela 1).

Considerando-se os valores de $\mathrm{Tb}$ obtidos a partir do método do menor desvio-padrão, para cada subperíodo, a duração média entre EME-MAT foi de $691^{\circ} \mathrm{C}$ dia, variando de $576^{\circ} \mathrm{C}$ dia a $792{ }^{\circ} \mathrm{C}$ dia. Para os subperíodos EME-IFLO, EME-FFLO e IFLO-FFLO, a duração média foi de $279^{\circ} \mathrm{C}$ dia; $444^{\circ} \mathrm{C}$ dia e $220^{\circ} \mathrm{C}$ dia respectivamente (Tabela 2 ).

Em trabalho desenvolvido por Adamsen e Coffelt (2005), avaliando a duração do subperíodo da semeadura ao início de floração, obtiveram-se valores entre $957^{\circ} \mathrm{C}$ dia e $1352^{\circ} \mathrm{C}$ dia. Ao comparar tais resultados aos obtidos para o subperíodo emergência-início de floração (EMEIFLO) (Tabela 2), constata-se acentuada diferença entre as constantes térmicas. Os resultados se embasam principalmente na amplitude dos valores de $\mathrm{Tb}$ utilizados para o cálculo, igual a $10,2^{\circ} \mathrm{C}$ nesse trabalho, e de $0{ }^{\circ} \mathrm{C}$ pelos referidos autores, e também pelo método empregado.

Analisando os valores dos coeficientes de variaçáo (CV) do ciclo de desenvolvimento em dias (Tabela 1) e em ${ }^{\circ} \mathrm{C}$ dia (Tabela 2), evidencia-se claramente o efeito da temperatura do ar sobre o desenvolvimento do crambe, pela reduçáo de mais de $50 \%$ dos $\mathrm{CV}$ para ${ }^{\circ} \mathrm{C}$ dia.

$\mathrm{Na}$ figura 3 tem-se a relação entre a temperatura média do ar e a duraçáo de cada fase de desenvolvimento, com as respectivas equaçóes de estimativa e coeficientes de determinação. Para a fase de EME-MAT, observa-se que mais $66 \%$ da variância da duração do referido período de desenvolvimento é explicada pela variância da temperatura do ar. Em relação aos demais, o subperíodo IFLOFFLO parece ser menos influenciado pela temperatura do ar, a qual explica $50 \%$ de sua variância.

Adamsen e Coffelt (2005), trabalhando com desenvolvimento de crambe (cv. Meyer), não obtiveram uma boa relação entre temperatura do ar e duração do período de semeadura a início da floraçáo. Conforme os autores, à medida que a semeadura foi realizada mais tardiamente, observou-se decréscimo na soma térmica para tal período 
Tabela 1. Duração do subperíodo emergência-maturação (EME-MAT) e subperíodos emergência-início de floração (EME-IFLO), emergência-fim de floração (EME-FFLO) e início de floração-fim de floração (IFLO-FFLO), em dias, para as diferentes datas de semeadura em 2009 e 2010. Temperatura média do ar para o subperíodo EME-MAT e subperíodo EME-IFLO e temperatura mínima absoluta ocorrida para o subperíodo EME-IFLO, em Frederico Westphalen (RS)

\begin{tabular}{|c|c|c|c|c|c|c|c|}
\hline \multirow[b]{2}{*}{ Data de semeadura } & \multicolumn{4}{|c|}{ Dias } & \multicolumn{2}{|c|}{ Tmed $\left({ }^{\circ} \mathrm{C}\right)$} & \multirow{2}{*}{$\begin{array}{c}\text { Tmin }\left({ }^{\circ} \mathrm{C}\right) \\
\text { EME- } \\
\text { IFLO }\end{array}$} \\
\hline & $\begin{array}{l}\text { EME- } \\
\text { MAT }\end{array}$ & $\begin{array}{l}\text { EME- } \\
\text { IFLO }\end{array}$ & $\begin{array}{l}\text { EME- } \\
\text { FFLO }\end{array}$ & $\begin{array}{l}\text { IFLO- } \\
\text { FFLO }\end{array}$ & $\begin{array}{l}\text { EME- } \\
\text { MAT }\end{array}$ & $\begin{array}{l}\text { EME- } \\
\text { IFLO }\end{array}$ & \\
\hline 13/5/2009 & 136 & 86 & 123 & 37 & 15,2 & 13,9 & $-2,7$ \\
\hline 28/5/2009 & 126 & 76 & 114 & 38 & 15,0 & 14,1 & $-2,7$ \\
\hline $11 / 6 / 2009$ & 127 & 67 & 107 & 40 & 15,8 & 14,5 & $-2,7$ \\
\hline $14 / 7 / 2009$ & 86 & 40 & 73 & 33 & 16,5 & 17,6 & 5,4 \\
\hline $31 / 7 / 2009$ & 90 & 42 & 74 & 32 & 18,3 & 17,7 & 5,4 \\
\hline $17 / 8 / 2009$ & 79 & 44 & 69 & 25 & 18,9 & 17 & 5,1 \\
\hline 3/9/2009 & 77 & 47 & 64 & 17 & 19,7 & 17,3 & 5,1 \\
\hline 23/6/2010 & 74 & 35 & 63 & 28 & 16,6 & 17,6 & 2,0 \\
\hline 8/7/2010 & 88 & 49 & 77 & 28 & 16,2 & 16,2 & 2,0 \\
\hline 29/7/2010 & 77 & 44 & 64 & 20 & 17,5 & 17,8 & 2,0 \\
\hline 29/8/2010 & - & 44 & 71 & 27 & - & - & - \\
\hline Média & 96 & 52 & 82 & 29 & 17,0 & 16,4 & 1,9 \\
\hline CV (\%) & 24,93 & 31,58 & 26,8 & 27,13 & 9,3 & 9,7 & 183,7 \\
\hline
\end{tabular}

Tabela 2. Constante térmica para o período emergência-maturação (EME-MAT), e subperíodos emergência-início de floração (EMEIFLO), emergência-fim de floração (EME-FFLO) e início de floração-fim de floração (IFLO-FFLO), para as diferentes datas de semeadura em 2009 e 2010. Valores de temperatura base inferior (Tb) foram obtidos pelo método do menor desvio-padrão, em Frederico Westphalen (RS)

\begin{tabular}{|lrccc|}
$\begin{array}{l}\text { Data de } \\
\text { semeadura }\end{array}$ & \multicolumn{4}{c}{ Constante térmica ( ${ }^{\circ} \mathrm{C}$ dia) } \\
\hline Tb & 9,5 & 10,2 & 10,8 & 10,0 \\
\hline $13 / 5 / 2009$ & 694,34 & 283,89 & 442,92 & 241,13 \\
\hline $28 / 5 / 2009$ & 693,71 & 262,27 & 444,87 & 256,55 \\
\hline $11 / 6 / 2009$ & 792,35 & 262,21 & 457,58 & 268,53 \\
\hline $14 / 7 / 2009$ & 653,51 & 275,06 & 431,84 & 206,82 \\
\hline $31 / 7 / 2009$ & 769,69 & 296,37 & 458,95 & 213,13 \\
\hline $17 / 8 / 2009$ & 724,74 & 283,7 & 481,50 & 245,33 \\
\hline $3 / 9 / 2009$ & 749,97 & 316,37 & 504,98 & 232,17 \\
\hline $23 / 6 / 2010$ & 576,46 & 248,5 & 402,57 & 209,16 \\
\hline $8 / 7 / 2010$ & 618,64 & 271,95 & 426,58 & 209,16 \\
\hline $29 / 7 / 2010$ & 632,94 & 313,28 & 421,76 & 149,2 \\
\hline 29/8/2010 & ---- & 251,24 & 413,93 & 187,8 \\
\hline Média & 690,64 & 278,62 & 444,32 & 219,91 \\
\hline CV (\%) & 10,15 & 8,22 & 6,79 & 15,38 \\
\hline & & & & \\
\hline
\end{tabular}

de desenvolvimento, sugerindo que outros fatores, tal como o fotoperíodo, poderia ser responsável pelo início da floração. Os resultados verificados para o subperíodo EME e IFLO (Tabela 2), da cultivar FMS-Brilhante, diferentemente ao observado por AdAmsen e Coffelt (2005), parecem não ter resposta ao fotoperíodo.

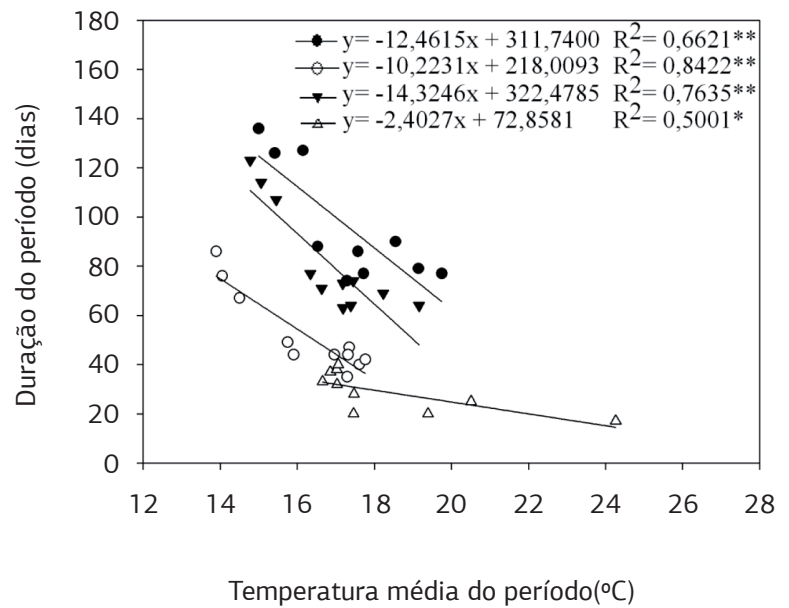

Figura 3. Relação entre a duração do período emergênciamaturação (• EME-MAT) e subperíodos emergência-início de floraçâo (o EME-IFLO), emergência-fim de floração ( $\boldsymbol{\nabla}$ EMEFFLO) e início de floração-fim de floração ( $\triangle$ IFLO-FFLO) com a temperatura média do ar para os respectivos períodos e subperíodos. ${ }^{*} \mathrm{e}^{* *}$ : Significativo pelo teste F, respectivamente, a $5 \%$ e $1 \%$ de probabilidade.

\section{CONCLUSÃO}

Temperatura basal inferior para subperíodo EME-MAT difere ligeiramente dos valores obtidos para os subperíodos EME-IFLO, EME-FFLO e IFLO-FFLO. Para cada um dos subperíodos, em função dos métodos utilizados, foram obtidos os valores de temperatura basal de $9,5^{\circ} \mathrm{C}$ e $8,9^{\circ} \mathrm{C}$ para EME-MAT, de $10,2^{\circ} \mathrm{C}$ e $9,6{ }^{\circ} \mathrm{C}$ para EMEIFLO, de $10,8^{\circ} \mathrm{C}$ e $10,1^{\circ} \mathrm{C}$ para EME-FFLO e $10,0^{\circ} \mathrm{C}$ e $9,2^{\circ} \mathrm{C}$ para IFLO-FFLO.

Por meio da temperatura do ar explica-se a maior parte da variância do ciclo de desenvolvimento da cultivar de crambe FMS Brilhante, com relação linear negativa. A partir da soma térmica são necessários de $576^{\circ} \mathrm{C}$ dia a 
$792^{\circ} \mathrm{C}$ dia para que a cultivar de crambe FMS Brilhante cumpra o período EME e MAT, com base na temperatura basal de $9,5^{\circ} \mathrm{C}$.

\section{AGRADECIMENTOS}

Ao Conselho Nacional de Desenvolvimento Científico e Tecnológico $(\mathrm{CNPq})$ pelo suporte financeiro e pela bolsa de iniciação científica.

\section{REFERÊNCIAS}

ADAMSEN, F.J., COFFELT, T.A. Plantig date effects on flowering, seed yield, and oil content of rape and crambe cultivars. Industrial Crops and Products, v.21, p.293-307, 2005.

ARNOLD, C.Y. The determination and significance of the base temperature in a linear heat unit system. Proceedings of the American Society for Horticultural Science, v.74, p.430-445, 1959.

BRUNINI, O.; LISBÃO, R.S.; BERNARDI, J.B.; FORNASIER, J.B.; PEDRO JÚNIOR, M.J. Temperatura-base para alface cultivar "White Boston", em um sistema de unidades térmicas. Bragantia, v.35, p.213-219, 1976.

DALMAGO, G.A.; CUNHA, G.R.; TOMM, G.O.; PIRES, J.L.F.; SANTI, A.; PASINATO, A.; SCHWEIG, E.; MÜLLER, A.L. Zoneamento agroclimático para a canola no Rio Grande do Sul. Passo Fundo: Embrapa Trigo, 2008. 35p.

EMBRAPA. Centro Nacional de Pesquisa de Solos. Sistema Brasileiro de Classificação de Solos. Rio de Janeiro: Embrapa-SPI, 1999. 412p.

FALASCA, S.L.; LAMAS, M.C.; CARBALLO, S.M.; ANSCHAU, A. Crambe abyssinica: An almost unknown crop with a promissory future to produce biodiesel in Argentina. International Journal of Hydrogen Energy, v.35, p.5808-5812, 2010.

FOWLER, J.L. Interaction of salinity and temperature on the germination of crambe. Agronomy Journal, v.83, p.169-172, 1991

GBUR, E.E.; THOMAS, G.L.; MILLER, F.R. Use of segmented regression in determination of the base-temperature in heat accumulation models. Agronomy Journal, v.71, p.949-953, 1979.

GILMORE Jr., E.C.; ROGERS, J.S. Heat units as a method of measuring maturity in corn. Agronomy Journal, v.50, p.611-615, 1958.

GOLZ, T. Crambe. Alternative Agriculture. North Dakota State University Extension Service, 1993 [cited 2010 Nov 10]. Available from: http://www.ext.nodak.edu/extnews.

HAWKINS, G.P.; DENG, Z.; KUBIK, T.J.; JOHNSONFLANAGAN, A.M. Characterization of freezing tolerance and vernalization in Vern-, a spring-type Brassica napus line derived from a winter cross. Planta, v.216, p.220-226, 2002.

KMEC, P.; WEISS, M.J.; MILBRATH, L.R.; SCHATZ, B.G.; HANZEL, J.; HANSON, B.K.; ERIKSMOEN, E.D. Growth analysis of crambe. Crop Science, v.38, p.108-112, 1998.

KNIGHTS, E.G. Crambe: A North Dakota case study. A Report for the Rural Industries Research and Development Corporation, RIRDC Publication No. W02/005, Kingston, 2002. 25p [cited 2010 Jul 16]. Available from: http://www.rirdc.gov.au.

LAZZERI, L.; LAPENTA, E.; SANTANGELO, E.; MALAGUTI, L.; VENTRELLA, D.; PINHEIRO, M. Crambe abyssinica Hochst ex R.E. Fries: agronomic performance and oil quality in three locations in Italy. Agricoltura Mediterranea, v.125, p.251-266, 1995.

LESSMAN, K.J.; MEIER, J.D. Agronomic evaluation of crambe as a source of oil. Crop Science, v.12, p.224-227, 1972.

MASSEY, J.H.; JELLUM, M.D. Effects of spring planting date and row spacing on the agronomic characteristics and chemical composition of crambe. Agronomy Journal, v.65, p.299-300, 1973.

MEIJER, W.J.M.; MATHIJSSEN, E.W.J. Analysis of crop performance in research on inulin, fibre and oilseed crops. Industrial Crops and Products, v.5, p.253-264, 1996.

MONTEITH, J.L.; ELSTON, J. Climatic constraints on crop production. In: FOWDEN, L.; MANSFIELD, T.; STODDART, J. (Ed.). Plant adaptation to environmental stress. London: Chapman \& Hall, 1996. p.3-18.

MORENO, J.A. Clima do Rio Grande do Sul. Porto Alegre: Secretaria da Agricultura, 1961. 41p.

MORRISON, M.J.; MCVETTY, P.B.E.; SHAYKEWICH, C.F. The determination and verification of a baseline temperature for the growth of Westar summer rape. Canadian Journal of Plant Science, v.69, p.455-464, 1989.

MÜLLER, L.; MANFRON, P.A.; MEDEIROS, S.L.P.; STRECK, N.A.; MITTELMMAN, A.; DOURADO NETO, D.; BANDEIRA, A.H.; MORAIS, K.P. Temperatura base inferior e estacionalidade de produção de genótipos diplóides e tetraplóides de azevém. Ciência Rural, v.39, p.1343-1348, 2009.

PEDRO JÚNIOR, M.J.; CAMARGO, M.B.P.; MORAES, A.V.C.; FELÍCIO, J.C.; CASTRO, J.L. Temperatura-base, grausdia e duração do ciclo para cultivares de triticale. Bragantia, v.63, p.447-453, 2004

PEZZOPANE, J.R.M.; PEDRO JÚNIOR, M.J.; CAMARGO, M.B.P.; FAZUOLI, L.C. Exigência térmica do café arábica cv. Mundo Novo no subperíodo florescimento-colheita. Ciência e Agrotecnologia, v.32, p.1781-1786, 2008.

RAPACZ, M. Frost resistance and cold acclimation abilities of spring-type oilseed rape. Plant Science, v.147, p.55-64, 1999. 
SOUZA, A.P.; SILVA, A.C.; LEONEL, S.; ESCOBEDO, J.F. Temperaturas basais e soma térmica para a figueira podada em diferentes épocas. Revista Brasileira de Fruticultura, v.31, p.314-322, 2009.

STRECK, N.A.; WEISS, A.; XUE, Q.; BAENZIGER, P.S. Improving predictions of developmental stages in winter wheat: a modified Wang and Engel model. Agricultural and Forest Meteorology, v.115, p.139-150, 2003.

TOEBE, M.; LOPES, S.J.; STORCK, L.; SILVEIRA, T.R.; MILANI, M.; CASAROTTO, G. Estimativa de plastocrono em crambe. Ciência Rural, v.40, p.793-799, 2010.
TOMM, G.O.; WIETHÖLTER, S.; DALMAGO, G.A.; SANTOS, H.P. Tecnologia para produção de canola no Rio Grande do Sul. Passo Fundo: Embrapa Trigo, 2009. 41 p. html. (Embrapa Trigo. Documentos Online, 113) [cited 2010 Jul 16]. Available from: http:/www.cnpt.embrapa.br/biblio/do/p_do113.htm.

WENT, F.W. The Effect of temperature on plant growth. Annual Review of Plant Physiology, v.4, p.347-362, 1953.

WHITE, G.A.; HIGGINS, J.J. Culture of crambe a new industrial oilseed crop. USDA, 1966. (Production Research Report n.95) 